\title{
Construction of Block Orthogonal Golay Sequences and Application to Channel Estimation of MIMO-OFDM Systems
}

\author{
Oh-Soon Shin, H. T. Kung, and Vahid Tarokh, Senior Member, IEEE
}

\begin{abstract}
In this paper, we construct a family of block orthogonal Golay sequences that have low peak-to-mean envelope power ratio (PMEPR) as well as blockwise orthogonal properties. We then present an application of the sequences to channel estimation of multiple-input multiple-output orthogonal frequency division multiplexing (MIMO-OFDM) systems. We compare the performance of the proposed algorithm with that of a frequency division multiplexing (FDM) piloting algorithm, and investigate the effect of co-channel interference (CCI) on the channel estimation performance.
\end{abstract}

Index Terms-Channel estimation, Golay sequences, multipleinput multiple-output (MIMO), orthogonal frequency division multiplexing (OFDM), peak-to-mean envelope power ratio (PMEPR).

\section{INTRODUCTION}

$\mathbf{O}$ RTHOGONAL frequency division multiplexing (OFDM) has received growing attention in wireless communications. Various forms of OFDM have been adopted in wireless standards, such as digital audio/video broadcasting, IEEE $802.11 \mathrm{a} / \mathrm{g}$, and IEEE 802.16 . For high data rate applications, OFDM offers a number of advantages including resistance to delay spread of wireless channel, frequency diversity combined with channel coding, and multiple access capability [1]. However, a major challenge of deploying OFDM is dealing with the relatively high peak-to-mean envelope power ratio (PMEPR) of the OFDM signal. A high PMEPR requires reducing the transmit power and efficiency of power amplifiers to lower their operating point. A desirable solution to this problem is to employ codes and sequences with low PMEPR property. In particular, Golay sequences that are invented in [2] are attractive, since their PMEPR is at most 2 [3].

On the other hand, the use of multiple transmit and receive antennas, called multiple-input multiple-output (MIMO), can provide enormous capacity enhancement of wireless systems. Recently, the combination of MIMO and OFDM, known as MIMO-OFDM, has attracted considerable attention as an emerging broadband wireless access technology. When a MIMO-OFDM system is implemented, accurate channel estimation is essential for coherent MIMO detection. Channel estimation usually utilizes pilot sequences that are known to

Paper approved by S. K. Wilson, the Editor for Multicarrier Modulation of the IEEE Communications Society. Manuscript received November 4, 2005 ; revised November 23, 2006. The work of O.-S. Shin was supported by the Soongsil University Research Fund.

O.-S. Shin was with the Division of Engineering and Applied Sciences, Harvard University, Cambridge, MA USA. He is now with Soongsil University, Seoul 156-743, Korea (e-mail: osshin@ssu.ac.kr).

H. T. Kung and V. Tarokh are with the Division of Engineering and Applied Sciences, Harvard University, Cambridge, MA 02138 USA (e-mail: kung@harvard.edu; vahid@deas.harvard.edu).

Digital Object Identifier 10.1109/TCOMM.2008.050492. the receiver. In order to facilitate channel estimation, the pilot sequences for the multiple transmit antennas are desired to be orthogonal to one another.

Since Golay sequences give low PMEPR, it is natural to use them for OFDM systems. This is particularly true for the pilot sequences of the MIMO-OFDM system. It must be noted that low PMEPR of the pilot sequences allows power boosting of pilot signals compared with data signals, improving the accuracy of channel estimation. In [4], pilot sequences for two transmit antennas have been designed to be orthogonal over each pair of two subcarriers. In this work, we introduce block orthogonality by extending this idea to the number of transmit antennas of $2^{n}$, and construct a family of Golay sequences that satisfy this property. Then we apply the sequences to channel estimation of MIMO-OFDM systems. Simulation results are provided to demonstrate the performance of the proposed channel estimation algorithm. In particular, we compare the performance of the proposed algorithm with that of a frequency division multiplexing (FDM) piloting algorithm taking co-channel interference (CCI) into consideration.

\section{Preliminaries}

We consider only pilot sequences for the channel estimation and the corresponding OFDM signal that is formed by a phase shift keying (PSK) modulation. The lowpass equivalent of the transmit OFDM signal $S_{\boldsymbol{s}}(t)$ constructed by a length $N$ sequence $s=\{s(i)\}_{i=0}^{N-1}$ defined over $\mathbb{Z}_{H}$ can be written as

$$
S_{\boldsymbol{s}}(t)=\sum_{i=0}^{N-1} \zeta^{s(i)} e^{j 2 \pi f_{i} t}
$$

where $\zeta \triangleq e^{j 2 \pi / H}, \zeta^{s(i)}$ is the $H$-PSK modulated symbol of $s(i)$, and $f_{i} \triangleq f_{0}+i \Delta f$ denotes the frequency of the $i$-th subcarrier. The frequency spacing $\Delta f$ should be an integer multiple of the OFDM symbol rate to maintain orthogonality among subcarriers. The PMEPR of the OFDM signal in (1) can be computed as [5]

$$
\operatorname{PMEPR}_{\boldsymbol{s}}=\sup _{t \in[0, T]} \frac{T\left|S_{\boldsymbol{s}}(t)\right|^{2}}{N} .
$$

Let $\boldsymbol{s}_{1}=\left\{s_{1}(i)\right\}_{i=0}^{N-1}$ and $\boldsymbol{s}_{2}=\left\{s_{2}(i)\right\}_{i=0}^{N-1}$ be distinct sequences with $s_{1}(i), s_{2}(i) \in \mathbb{Z}_{H}$. Define the aperiodic autocorrelation of $\boldsymbol{s}_{\ell}$ at displacement $d$ as $C_{\boldsymbol{s}_{\ell}}(d) \triangleq$ $\sum_{0 \leq i, i+d<N} \zeta^{s_{\ell}(i)-s_{\ell}(i+d)}$. Then, the sequences $s_{1}$ and $s_{2}$ are called a Golay complementary pair over $\mathbb{Z}_{H}$ if $C_{\boldsymbol{s}_{1}}(d)+$ $C_{\boldsymbol{s}_{2}}(d)=0$ for any $d \neq 0$. Any sequence which is a member of a Golay complementary pair is called a Golay sequence [5]. It is well known that the PMEPR of any Golay sequence is at most 2 [3], which makes it attractive for OFDM systems. A 
systematic way of constructing Golay sequences of length $2^{n}$ defined over $\mathbb{Z}_{2^{h}}$ has been developed by Davis and Jedwab [5]. For completeness, we provide the main Theorem of Davis and Jedwab here.

Theorem 1: (Davis and Jedwab [5]) Suppose $N=2^{n}$ and $H=2^{h}$. Let $\left(x_{1}^{i} x_{2}^{i} \cdots x_{n}^{i}\right)$ be the binary representation of the integer $i, 0 \leq i<N$, and let $x_{0}^{i}=1$ for any $i$. For any permutation $\omega$ of the symbols $\{1,2, \cdots, n\}$ and for any $c_{k} \in \mathbb{Z}_{H}, \quad k=0,1, \cdots, n$, the sequence $\boldsymbol{g}=\{g(i)\}_{i=0}^{N-1}$ given by

$$
g(i)=2^{h-1} \sum_{k=1}^{n-1} x_{\omega(k)}^{i} x_{\omega(k+1)}^{i}+\sum_{k=0}^{n} c_{k} x_{k}^{i}, \quad 0 \leq i<N
$$

is a Golay sequence over $\mathbb{Z}_{H}$ of length $N$.

Theorem 1 states that Golay sequences are given as cosets of the first-order Reed-Muller codes [6] in the second-order Reed-Muller codes with coset representatives $\left\{\sum_{k=1}^{n-1} x_{\omega(k)}^{i} x_{\omega(k+1)}^{i}: 0 \leq i<N\right\}$, and provides a total of $2^{h(n+1)} n ! / 2$ Golay sequences of length $2^{n}$ over $\mathbb{Z}_{2^{h}}$.

\section{Block Orthogonal Golay Sequences}

We first define the block-M orthogonality of sequences over PSK modulation.

Definition 1: Let $s_{1}, s_{2}, \cdots, s_{L}$ be distinct sequences over $\mathbb{Z}_{2^{h}}$, where the $\ell$-th sequence $\boldsymbol{s}_{\ell}=\left\{s_{\ell}(i)\right\}_{i=0}^{N-1}$ is a sequence of length $N$. Assume that $N$ is divisible by $M$. We define the followings.

- Any two distinct sequences $\boldsymbol{s}_{\ell}$ and $\boldsymbol{s}_{k}(\ell \neq k)$ are orthogonal, if $\sum_{i=0}^{N-1} \zeta^{s_{\ell}(i)-s_{k}(i)}=0$, where $\zeta \triangleq e^{j \frac{2 \pi}{2^{h}}}$.

- Any two distinct sequences $\boldsymbol{s}_{\ell}$ and $\boldsymbol{s}_{k}(\ell \neq k)$ are block-M orthogonal, if the consecutive disjoint sections of the sequences with length $M$ are orthogonal, i.e., $\sum_{i=0}^{M-1} \zeta^{s_{\ell}(j M+i)-s_{k}(j M+i)}=0$ for all $0 \leq j<N / M$.

- The $L$ sequences $s_{1}, s_{2}, \cdots, s_{L}$ form a set of block-M orthogonal sequences, if any pair of two sequences are block- $M$ orthogonal.

The following Lemma provides a condition on which a pair of first-order Reed-Muller codes are orthogonal.

Lemma 1: Assume $c_{k}, c_{k}^{\prime} \in \mathbb{Z}_{2^{h}}$ for $k=1,2, \cdots, n$. Let $\left(x_{1}^{i} x_{2}^{i} \cdots x_{n}^{i}\right)$ be the binary representation of the integer $i, 0 \leq i<N$. Consider sequences $s_{1}=\left\{s_{1}(i)\right\}_{i=0}^{N-1}$ and $\boldsymbol{s}_{2}=\left\{s_{2}(i)\right\}_{i=0}^{N-1}$ of length $N=2^{n}$ over $\mathbb{Z}_{2^{h}}$, generated by first-order Reed-Muller codes:

$$
\begin{aligned}
& s_{1}(i)=\sum_{k=1}^{n} c_{k} x_{k}^{i}, \\
& s_{2}(i)=\sum_{k=1}^{n} c_{k}^{\prime} x_{k}^{i}, \quad 0 \leq i<N .
\end{aligned}
$$

Then $s_{1}$ and $s_{2}$ are orthogonal, if and only if $c_{k}-c_{k}^{\prime}=2^{h-1}$ for at least one $k$ in $1 \leq k \leq n$.
Proof: We can check the orthogonality between $s_{1}$ and $\boldsymbol{s}_{2}$ as

$$
\begin{aligned}
& \sum_{i=0}^{N-1} \zeta^{s_{1}(i)-s_{2}(i)}=\sum_{i=0}^{N-1} \zeta^{\sum_{k=1}^{n}\left(c_{k}-c_{k}^{\prime}\right) x_{k}^{i}} \\
& =\sum_{\left(x_{1}^{i} \cdots x_{n}^{i}\right) \in \mathbf{Z}_{2}^{n}} \zeta^{\sum_{k=1}^{n}\left(c_{k}-c_{k}^{\prime}\right) x_{k}^{i}} \\
& =\sum_{\left(x_{2}^{i} \cdots x_{n}^{i}\right) \in \mathbf{Z}_{2}^{n-1}} \zeta^{\sum_{k=2}^{n}\left(c_{k}-c_{k}^{\prime}\right) x_{k}^{i}+}
\end{aligned}
$$

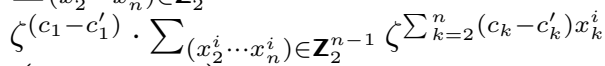

$$
\begin{aligned}
& =\left(1+\zeta^{\left(c_{1}-c_{1}^{\prime}\right)}\right)^{2} \cdot \sum_{\left(x_{2}^{i} \cdots x_{n}^{i}\right) \in \mathbf{Z}_{2}^{n-1}} \zeta^{\sum_{k=2}^{n}\left(c_{k}-c_{k}^{\prime}\right) x_{k}^{i}} \\
& =\cdots \\
& =\prod_{k=1}^{n}\left(1+\zeta^{\left(c_{k}-c_{k}^{\prime}\right)}\right),
\end{aligned}
$$

which is zero, if and only if $c_{k}-c_{k}^{\prime}=2^{h-1}$ for at least one $k$ in $1 \leq k \leq n$.

Using Lemma 1, we now present main theorem of this paper that shows how to construct block $-M$ orthogonal Golay sequences.

Theorem 2: Suppose $N=2^{n}, H=2^{h}$, and $M=2^{m}$ $(m \leq n)$. Let $\left(x_{1}^{i} x_{2}^{i} \cdots x_{n}^{i}\right)$ be the binary representation of the integer $i, 0 \leq i<N$, and let $x_{0}^{i}=1$ for any $i$. For any permutation $\omega$ of the symbols $\{1,2, \cdots, n\}$ and for any $c_{k} \in \mathbb{Z}_{H}, 0 \leq k \leq n-m$, define a sequence $\boldsymbol{r}=\{r(i)\}_{i=0}^{N-1}$ as

$$
r(i)=2^{h-1} \sum_{k=1}^{n-1} x_{\omega(k)}^{i} x_{\omega(k+1)}^{i}+\sum_{k=0}^{n-m} c_{k} x_{k}^{i},
$$

which is a Golay sequence in (3) with $c_{k}=0$ for $n-m<$ $k \leq n$. Define $H / 2$ sets of sequences $\boldsymbol{G}_{\ell}, 1 \leq \ell \leq H / 2$ as

$$
\begin{aligned}
\boldsymbol{G}_{\ell} \triangleq & \left\{\left\{r(i)+\sum_{k=n-m+1}^{n} c_{k} x_{k}^{i}\right\}_{i=0}^{N-1} \mid\right. \\
& \left.c_{k} \in\{\ell-1, \ell-1+H / 2\}, n-m<k \leq n\right\} .
\end{aligned}
$$

Then each set $\boldsymbol{G}_{\ell}$ contains $M$ sequences and forms a set of block-M orthogonal Golay sequences.

Proof: From Theorem 1, any sequence in $\boldsymbol{G}_{\ell}$ is a Golay sequence. Furthermore, any pair of sequences in $\boldsymbol{G}_{\ell}$ are block$M$ orthogonal, since $\boldsymbol{r}$ is a term common to all sequences in $\boldsymbol{G}_{\ell}$ and the sequences in the second term are block- $M$ orthogonal by Lemma 1 . Hence, $\boldsymbol{G}_{\ell}$ forms a set of block- $M$ orthogonal sequences.

Corollary 1: The $2^{n} n$ ! binary Golay sequences can be partitioned into $2^{n-m} n$ ! disjoint sets of block-M orthogonal sequences, with each set containing $M$ Golay sequences.

Theorem 2 gives $H / 2$ sets of block $-M$ orthogonal Golay sequences for each given set of $\omega$ and $c_{k}, 0 \leq k \leq n-m$. It must be noted that the first component $\boldsymbol{r}$ of each sequence is common to all the sequences in each set, whereas the second block- $M$ orthogonal component $\sum_{k=n-m+1}^{n} c_{k} x_{k}^{i}$ is periodic with period $M$, since it depends only on the $m$ least significant bits of $i$. For the binary $(h=1)$ case, Corollary 1 shows that any Golay sequence in (3) belongs to a certain set of block- $M$ orthogonal Golay sequences, since the sequences in (7) cover all the Golay sequences in (3). 


\section{Channel Estimation Algorithm for MIMO-OFDM SYSTEMS}

In this section, we propose a channel estimation algorithm for a MIMO-OFDM system that exploits the block orthogonality of the Golay sequences constructed in Section III. The MIMO-OFDM system is equipped with $N$ subcarriers, $M$ transmit antennas, and $K$ receive antennas. Each of the block- $M$ orthogonal Golay sequences within a set defined in Theorem 2 is assigned to one of $M$ transmit antennas, to form a pilot OFDM symbol as in (1). Specifically, according to the sequence definition of Theorem 2, we can describe the pilot sequence $\boldsymbol{s}_{v}$ of the $v$-th transmit antenna as $s_{v}(i)=$ $r(i)+\sum_{k=n-m+1}^{n} c_{k, v} x_{k}^{i}, 0 \leq i<N$, where $c_{k, v}, n-m<$ $v \leq n$ are coefficients specific to the $v$-th transmit antenna. We assume that the length $N$ of each sequence is the same as the number of subcarriers, and that all the subcarriers are used to transmit the pilot signals. When null subcarriers are employed, parts of the sequence need to be nulled, which may degrade the PMEPR property of Golay sequences. In [7], it has been empirically shown that we can still achieve similar PMEPR to the original sequence, if the fraction of null subcarriers is small, which is usually the case. Each transmit antenna sends $Q$ periods of the pilot symbol with a cyclic prefix attached in the beginning.

Assuming that transmit signals from $M$ transmit antennas are synchronous at the receiver, the received pilot signal $y_{u}(q, i)$ at the $u$-th receive antenna in the $i$-th subcarrier during the $q$-th OFDM symbol can be expressed as

$$
\begin{aligned}
y_{u}(q, i)= & \sqrt{P} \cdot \sum_{v=1}^{M} h_{u v}(i) \alpha_{v}(i)+\eta_{u}(q, i), \\
& 1 \leq u \leq K, 0 \leq i<N, 0 \leq q<Q
\end{aligned}
$$

where $P$ is the average received power from each transmit antenna, $h_{u v}(i)$ is the channel response at the $u$-th receive antenna in the $i$-th subcarrier of the $v$-th transmit antenna with the average power normalized to $E\left[\left|h_{u v}(i)\right|^{2}\right]=1, \alpha_{v}(i) \triangleq$ $\zeta^{s_{v}(i)}$ denotes the $H$-PSK modulated symbol of $s_{v}(i)$, and $\eta_{u}(q, i)$ denotes the additive white Gaussian noise (AWGN) with the variance $\sigma^{2}$. In (8), it is assumed that the channel does not vary during the channel estimation period of $Q$ OFDM symbols. The channel estimation problem at the receiver is to estimate $K M$ sets of channels $\left\{h_{u v}(i): 0 \leq i<N\right\}, 1 \leq$ $u \leq K, 1 \leq v \leq M$ from the signal in (8). We drop the subscript $u$ hereafter, since the same algorithm can be applied to each receive antenna.

As the first step of the channel estimation, we enhance the signal-to-noise ratio (SNR) of the received signal by averaging the signal in (8) over $Q$ periods as

$$
\begin{aligned}
y(i) & \triangleq \frac{1}{Q} \sum_{q=0}^{Q-1} y(q, i) \\
& =\sqrt{P} \cdot \sum_{v=1}^{M} h_{v}(i) \alpha_{v}(i)+\eta(i), \quad 0 \leq i<N
\end{aligned}
$$

where $\eta(i) \triangleq \frac{1}{Q} \sum_{q=0}^{Q-1} \eta(q, i)$ has variance $\sigma^{2} / Q$. The received signal suffers from mutual interference among different transmit antennas as well as the AWGN. As proposed in [4] for $M=2$, we can exploit the block- $M$ orthogonality of the pilot sequences to suppress the interference, assuming that channel variation is minimal over each block of $M$ adjacent subcarriers.

To make the following block processing easier, we take off the common component of the pilot sequences by multiplying the complex conjugate of modulated sequence $\left\{\zeta^{r(i)}\right\}_{i=0}^{N-1}$ to (9) as

$$
\begin{aligned}
z(i) & \triangleq y(i) \zeta^{-r(i)} \\
& =\sqrt{P} \cdot \sum_{v=1}^{M} h_{v}(i) \beta_{v}(i)+w(i), \quad 0 \leq i<N
\end{aligned}
$$

where $\beta_{v}(i) \triangleq \zeta^{s_{v}(i)-r(i)}=\zeta^{\sum_{k=n-m+1}^{n} c_{k, v} x_{k}^{i}}$ defines the modulated sequence corresponding to the transmit antennaspecific component of the sequence $\boldsymbol{s}_{v}$, and $w(i) \triangleq \eta(i) \zeta^{-r(i)}$. Note that $\beta_{v}(i)$ repeats every block of $M$ subcarriers, i.e., $\beta_{v}(i)=\beta_{v}(\ell M+i), 0 \leq i<M, 0 \leq \ell<N / M$, as discussed in Section III. Therefore, under the assumption that channel variation is minimal over each block of subcarriers, (10) can be approximated to a vector form in terms of the channel at the center of each block as

$$
\mathbf{Z}_{\ell} \approx \sqrt{P} \cdot \mathbf{B} \mathbf{H}_{\ell}+\mathbf{W}_{\ell}, \quad 0 \leq \ell<N / M
$$

where

$$
\begin{aligned}
& \mathbf{Z}_{\ell} \triangleq[z(\ell M) z(\ell M+1) \cdots z(\ell M+M-1)]^{T}, \\
& \mathbf{W}_{\ell} \triangleq[w(\ell M) w(\ell M+1) \cdots w(\ell M+M-1)]^{T} \text {, } \\
& \mathbf{B} \triangleq\left[\begin{array}{cccc}
\beta_{1}(0) & \beta_{2}(0) & \cdots & \beta_{M}(0) \\
\beta_{1}(1) & \beta_{2}(1) & \cdots & \beta_{M}(1) \\
\beta_{1}(2) & \beta_{2}(2) & \cdots & \beta_{M}(2) \\
\vdots & \vdots & \vdots & \vdots \\
\beta_{1}(M-1) & \beta_{2}(M-1) & \cdots & \beta_{M}(M-1)
\end{array}\right]
\end{aligned}
$$

and $\mathbf{H}_{\ell}$ is an $M \times 1$ channel vector at the center frequency of the $\ell$-th subcarrier block, i.e., at $f_{\ell M+(M-1) / 2}$. The $v$-th element of $\mathbf{H}_{\ell}$ is $h_{v}\left(\ell M+\frac{M-1}{2}\right)$, which is the channel response at $f_{\ell M+(M-1) / 2}$. Note that the matrix $\mathbf{B}$ is a Hadamard matrix [6] that is common to all the blocks, since $\beta_{v}(i)$ repeats every block. Since $\mathbf{B}^{H} \mathbf{B}=M \cdot \mathbf{I}$, we observe that an estimate $\tilde{\mathbf{H}}_{\ell}$ of the channel vector $\mathbf{H}_{\ell}$ can be derived from (11) as

$$
\tilde{\mathbf{H}}_{\ell}=\frac{1}{M \sqrt{P}} \mathbf{B}^{H} \mathbf{Z}_{\ell}=\mathbf{H}_{\ell}+\frac{1}{M \sqrt{P}} \mathbf{B}^{H} \mathbf{W}_{\ell}, \quad 0 \leq \ell<N / M
$$

which provides channel estimates for every block of $M$ subcarriers. To obtain full channel estimates for all subcarriers, we interpolate the block estimates for each transmit antenna as

$$
\begin{aligned}
& \left\{\left(f_{i}, \hat{h}_{v}(i)\right): 0 \leq i<N\right\}= \\
& \mathcal{I}\left(\left\{\left(f_{\ell M+(M-1) / 2}, \tilde{h}_{v}\left(\ell M+\frac{M-1}{2}\right)\right): 0 \leq \ell<N / M\right\}\right), \\
& \quad 1 \leq v \leq M
\end{aligned}
$$

where $\mathcal{I}(\cdot)$ denotes an interpolation function from a set of block estimates to an expanded set corresponding to the channel estimates for all subcarriers. The estimates in (16) may be noisy due to the interpolation error as well as the residual interference and noise. To improve the quality of the estimates, we apply a lowpass filter to the estimates in (16), which converts the estimates $\hat{h}_{v}(i)$ in (16) to the time domain, 


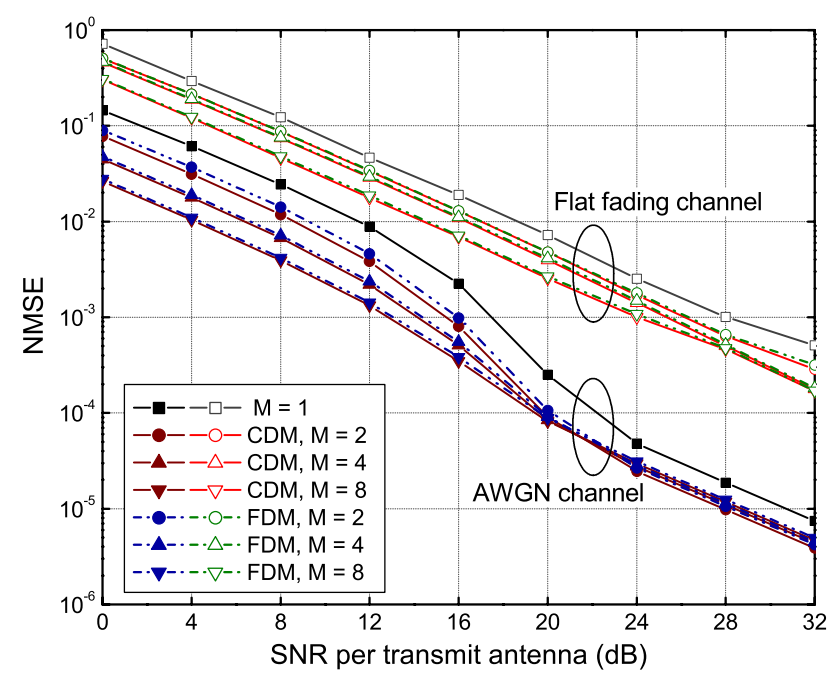

Fig. 1. NMSE performance in AWGN and flat fading channels without CCI for various number of transmit antennas $M$.

takes only the first $W$ samples of the time-domain impulse responses, and reconverts them to the frequency domain [4].

It must be noted that the proposed algorithm has been derived under the assumption that the channel variation is negligible across subcarriers within each block of size $M$. Thus, the proposed algorithm must be primarily suitable for indoor environments where frequency selectivity is not severe. The frequency selectivity of the channel will deteriorate the performance due to the loss of orthogonality among received pilot sequences. The performance degradation will be more significant for larger block size and/or larger subcarrier spacing.

\section{Channel Estimation Performance}

In this section, we evaluate the performance of the channel estimation algorithm proposed in Section IV. The number of OFDM subcarriers is assumed to be $N\left(=2^{n}\right)=64$. We let the pilot sequences for $M=2^{m}$ transmit antennas be the binary block $-M$ orthogonal Golay sequences constructed with $\omega(i)=i, 0 \leq i<N$, and $c_{k}=0,0 \leq k \leq n-m$. We use two indoor channel models used in [8], which are referred to as channel $\mathrm{A}$ and channel $\mathrm{B}$. The normalized multipath intensity profiles of channel $\mathrm{A}$ and channel $\mathrm{B}$ are given by $\{0.9981,0.0019\}$ and $\{0.9410,0.0573,0,0.0017\}$, respectively, with tap spacing of the OFDM sample duration. Every path of the channel is assumed to follow an independent complex Gaussian distribution. Note that channel B stands for a more frequency-selective channel than channel $\mathrm{A}$. We do not consider nonsample-spaced channels considered in [9], under the assumption that channel impulse responses are strictly confined within the cyclic prefix. This assumption is reasonable since the delay spread of indoor channels is usually small. The period of transmitted pilot sequences is taken to be $Q=2$. Both the length of cyclic prefix and the window length $W$ of the low pass filter are set to 10 . The linear interpolation is assumed to be employed in (16). We compare the performance of the proposed channel estimation algorithm with another algorithm. In the algorithm, pilot sequences

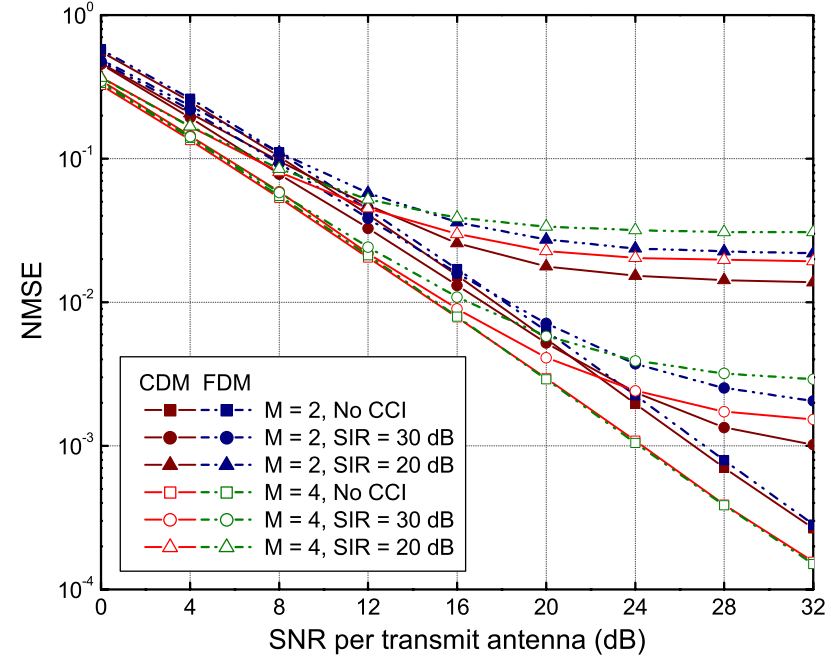

Fig. 2. NMSE performance in channel A for the number of transmit antennas $M=2$ and 4

for $M$ transmit antennas are transmitted in the frequency division fashion: a length $N / M$ Golay sequence for the $v$-th transmit antenna is transmitted on $(\ell M+v-1)$-th subcarriers $(0 \leq \ell<N / M)$. The estimates for the $N / M$ subcarriers are interpolated over all subcarriers, and low pass filtering is applied as in the proposed algorithm. This algorithm will be referred to as the FDM algorithm, while the proposed algorithm as code division multiplexing (CDM) algorithm. We measure the performance of the channel estimation algorithm using the normalized mean square error (NMSE) defined as

$$
\mathrm{NMSE} \triangleq \frac{1}{M} \sum_{v=1}^{M} \frac{\left\|\mathbf{H}_{v}-\hat{\mathbf{H}}_{v}\right\|^{2}}{\left\|\mathbf{H}_{v}\right\|^{2}} .
$$

The NMSE performance in AWGN and flat fading channels is shown in Fig. 1, when the CCI is not considered. The channel estimation algorithm is shown to work well for various numbers of transmit antennas $M$, in that the NMSE keeps decreasing with the SNR per transmit antenna, $P / \sigma^{2}$. In most of the SNR range in Fig. 1, the performance is shown to improve as $M$ increases. The reason is that larger $M$ yields higher averaging effect in obtaining the sample points of interpolation and thus less interpolation noise, when the channel is constant across the subcarriers as in the AWGN and flat fading channels. In most cases, the CDM algorithm is shown to slightly outperform the FDM algorithm.

Fig. 2 illustrates the NMSE performance for $M=2$ and $M=4$ in channel A. For both $M=2$ and $M=4$, the CDM and FDM algorithms provide comparable performance, when CCI is absent. However, when a CCI is present with the signal-to-interference ratio $(\mathrm{SIR})=30 \mathrm{~dB}$ or $20 \mathrm{~dB}$, the $\mathrm{CDM}$ algorithm is shown to significantly outperform the FDM algorithm especially in the high SNR region. This gain comes from the inherent CCI averaging capability of the CDM. The NMSE performance for channel B is depicted in Fig. 3. When the CCI is absent, the CDM algorithm is shown to be worse than the FDM algorithm. The reason for this is that the orthogonality among pilot sequences of $M$ transmit antennas is not preserved in channel $\mathrm{B}$, where the channel variation 


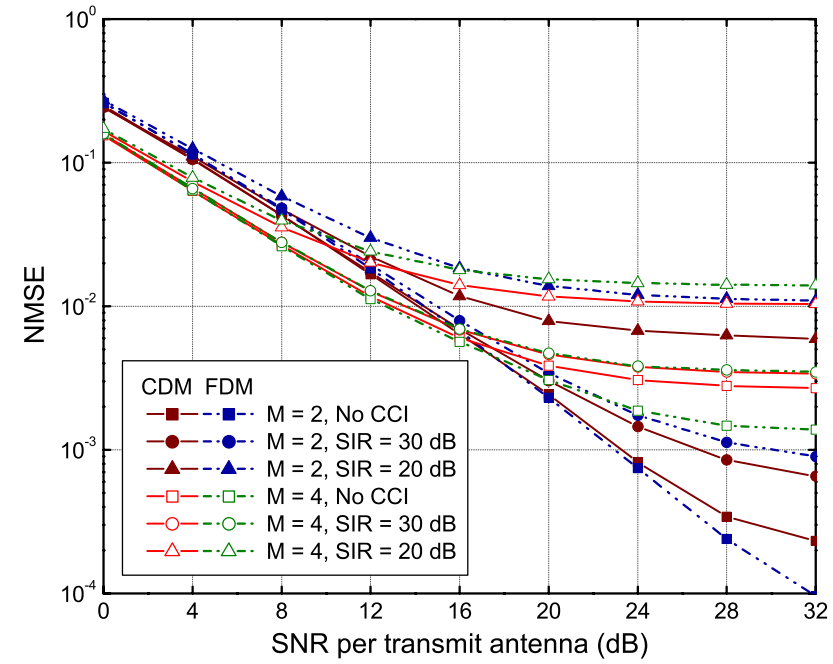

Fig. 3. NMSE performance in channel B for the number of transmit antennas $M=2$ and 4 .

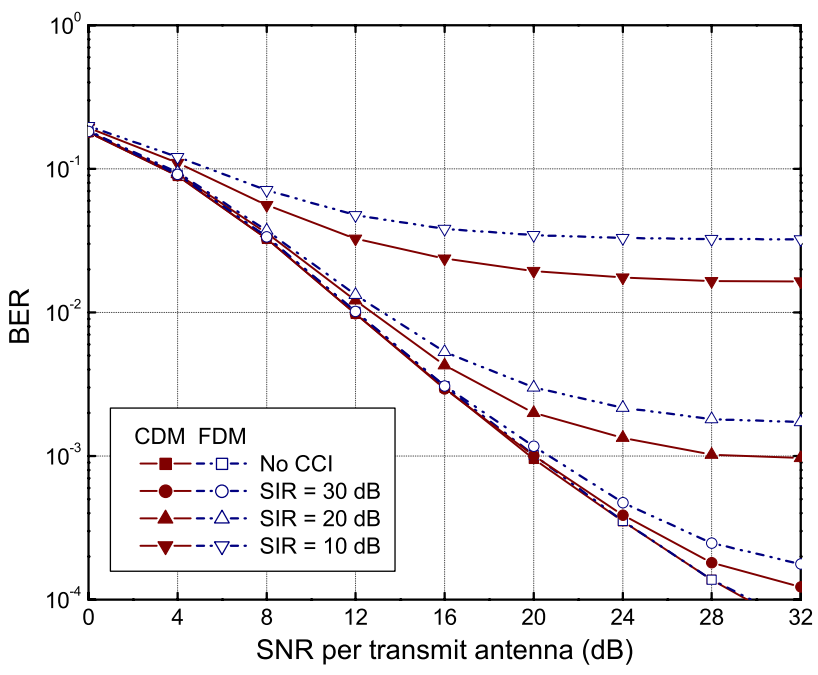

Fig. 4. BER performance of a $2 \times 2$ MIMO system in channel $A$, when uncoded QPSK is employed.

within each block of subcarriers is severer than in channel A. The mutual interference among transmit antennas yields an error floor, and it is more significant for a larger block size $M$. When a CCI is present, however, the CDM algorithm is shown to outperform the FDM algorithm. This implies that the CCI averaging effect of the CDM dominates that of the orthogonality loss in the presence of CCI.

Fig. 4 shows the impact of channel estimators on the bit error rate (BER) performance of a $2 \times 2$ MIMO system in channel A, when uncoded QPSK modulation is employed.
The receiver is assumed to detect transmit data using the VBLAST algorithm based on the MMSE detection [10]. This figure verifies that lower NMSE of the CDM algorithm indeed results in lower BER when a CCI is present. The improvement in the BER of the CDM algorithm over the FDM algorithm is shown to be more significant at lower SIR.

\section{CONCLUSIONS}

In this paper, we have defined and constructed a family of block orthogonal Golay sequences. It has been found that each sequence in a set of block orthogonal Golay sequences can be decomposed into two sequences; one component sequence is common to all the sequences in the set, whereas the other is specific to a sequence and repeats in every block. We have also applied block orthogonal Golay sequences to a channel estimation of MIMO-OFDM systems based on CDM of pilot sequences. Through simulation, we have compared the performance of the proposed algorithm with that of an algorithm based on the FDM of pilot sequences, in fading channels as well as in an AWGN channel. The proposed algorithm is shown to be less sensitive to the CCI compared with the FDM based algorithm. Hence, the proposed algorithm is expected to improve the channel estimation performance near cell boundaries of a cellular system.

\section{REFERENCES}

[1] R. V. Nee and R. Prasad, OFDM for Wireless Multimedia Communications. London: Artech House, 2000.

[2] M. J. E. Golay, "Complementary series," IRE Trans. Inform. Theory, vol. 7, pp. 82-87, Apr. 1961.

[3] B. M. Popović, "Synthesis of power efficient multitone signals with flat amplitude spectrum," IEEE Trans. Commun., vol. 39, pp. 1031-1033, July 1991.

[4] O.-S. Shin, A. Chan, H. T. Kung, and V. Tarokh, "Design of an OFDM cooperative space-time diversity system," IEEE Trans. Veh. Technol., vol. 56, pp. 2203-2215, July 2007.

[5] J. A. Davis and J. Jedwab, "Peak-to-mean power control in OFDM, Golay complementary sequences, and Reed-Muller codes," IEEE Trans. Inform. Theory, vol. 45, pp. 2397-2417, Nov. 1999.

[6] F. J. MacWilliams and N. J. A. Sloane, The Theory of Error-Correcting Codes. The Netherlands: Elsevier Science Publishers B.V., 1977.

[7] V. P. G. Jimenez, "Study and implementation of complementary Golay sequences for PAR reduction in OFDM signals," White Paper, Technische Universiteit Eindhoven, Dec. 2001.

[8] European Telecommunications Standards Institute (ETSI), "Universal mobile telecommunications system (UMTS): Selection procedures for the choice of radio transmission technologies for the UMTS," Technical Report 101.112, V3.2.0, Apr. 1998.

[9] O. Edfors, M. Sandell, J.-J. van de Beek, S. K. Wilson, and P. O. Börjesson, "OFDM channel estimation by singular value decomposition," IEEE Trans. Commun., vol. 46, pp. 931-939, July 1998.

[10] P. W. Wolniansky, G. J. Foschini, G. D. Golden, and R. A. Valenzuela, "V-BLAST: An architecture for realizing very high data rates over the rich-scattering wireless channel," in Proc. URSI Inter. Symp. Signals, Syst., Electronics, Sep.-Oct. 1998, pp. 295-300. 